\title{
GESTIÓN DE LA TUTORÍA TELEMÁTICA EN EDUCACIÓN A DISTANCIA
}

Ing. Jorge E. Grau*

Lic. María Irma Marabotto**

C.C. Estela N. Muelas ${ }^{* * *}$

\section{Resumen}

Este trabajo presenta una aplicación experimental de tutoría telemática mediante correo electrónico e Internet, realizada durante 1999 con los alumnos de la Primera Convocatoria del Curso Universitario de Postgrado en Educación a Distancia que se dictó en la República Argentina en la Universidad CAECE junto con la Fundación para el Desarrollo de los Estudios Cognitivos, FUNDEC.

Se analiza esta experiencia pedagógico-didáctica, desarrollada durante la primera parte del Curso, entre abril y junio de 1999, con casi cien alumnos distribuidos en más de $2.800 .000 \mathrm{~km} 2$, a la luz de las posibilidades de las herramientas informáticas utilizadas.

\section{Introducción}

Esta es una aplicación experimental de lo que se conoce como tutoría telemática, realizada mediante correo electrónico e Internet en 1999 con los alumnos de la Primera Convocatoria del Curso Universitario de Postgrado en Educación a Distancia que se dictó en la República Argentina, en el marco del Convenio de Colaboración Académica entre la Universidad CAECE y la Fundación para el Desarrollo de los Estudios Cognitivos, FUNDEC.

La Universidad CAECE, de acuerdo con la reglamentación vigente en el ámbito universitario, tuvo a su cargo la certificación del mismo, y FUNDEC fue la responsable de su organización y coordinación. El Curso estuvo dirigido por el Dr. Lorenzo García Aretio, Profesor Titular de Teoría de la Educación (Educación a Distancia) de la Facultad de Educación y ex-Director del Instituto Universitario de Educación a Distancia de la Universidad Nacional de Educación a Distancia (UNED) de España. Actualmente, el Dr. Lorenzo García Aretio es el Titular de la Cátedra UNESCO en Educación a Distancia.

Los destinatarios de este curso fueron profesionales, profesores o formadores, responsables de diseñar, gestionar y/o evaluar programas, quienes ya estaban implicados en procesos de enseñanza a distancia, que preveían que podían estarlo, o que entendían que saber sobre el tema les podía abrir nuevos caminos profesionales. El acceso al Curso de Postgrado implicaba una formación de nivel superior y experiencia en la docencia y en la gestión de proyectos educativos. A su vez, fue requisito obligatorio tener acceso a una cuenta de correo electrónico y a Internet.

\section{Las condiciones}

Hoy una línea telefónica y la computadora nos permiten contactarnos con otros usuarios, tanto a nivel nacional como internacional e intercambiar programas u obtener información, y en la mayoría de los casos a cualquier hora del día. El correo electrónico -Email- permite un fluido y rápido intercambio, ya que es un medio de comunicación persona-a-persona. A su vez, dentro de los distintos servicios que provee Internet, una Página Web es un sistema de información con las siguientes características: 1) es hipermedial: combina información hipertextual y recursos multimediales, 2) distribuido: con millones de servidores en todo el mundo, y 3 ) colaborativo: cualquiera puede agregar información. 
En la Enseñanza a Distancia las comunicaciones por computadora se orientan, prioritariamente, a las tareas de tutoría, es decir, de intercomunicación tutor-alumno, pero también pueden utilizarse para la intercomunicación de los alumnos entre sí. A través de estos recursos se pueden materializar: a) videoconferencias, b) video, c) audio, y d) transferencia de archivos, de las manera que más convenga al proceso de enseñanza-aprendizaje a desarrollar en la modalidad a distancia.

En este ámbito, el Correo Electrónico y la Página Web podrían configurar una reunión de un grupo humano, donde el docente puede vincularse con varios alumnos situados en lugares diferentes, y los alumnos también pueden vincularse entre sí. A la luz de estas herramientas informáticas se analiza la experiencia de tutoría telemática, desarrollada entre abril y junio de 1999 -durante la primera parte del Curso- con casi cien alumnos, distribuidos en más de $2.800 .000 \mathrm{~km}^{2}$.

\section{Los alumnos}

Esta convocatoria -organizada originariamente para la República Argentina- matriculó 98 alumnos; casi en su totalidad eran graduados universitarios, salvo algunos profesores egresados de institutos terciarios, aceptados por sus antecedentes en educación a distancia. Es interesante destacar que dicha convocatoria también incorporó dos alumnos que residían fuera del país, un graduado universitario del Uruguay y un graduado universitario argentino, residente en EE.UU.

En la matrícula, la procedencia profesional de los alumnos fue variada. Hubo graduados universitarios -ingenieros, computadores científicos, licenciados en economía, contadores, licenciados en ciencias de la educación-y profesores de enseñanza secundaria en diversas disciplinas. La distribución territorial de los alumnos por el país fue la siguiente (Tabla $\mathrm{N}^{\mathrm{o}} 1$ ):

\section{Tabla $\mathbf{N}^{0} 1$ : Distribución territorial de los alumnos}

\begin{tabular}{|l|c|}
\hline Distrito & Cursantes $\mathbf{1 9 9 9}$ \\
\hline Capital Federal & 53 \\
\hline Gran Buenos Aires $(50 \mathrm{~km})$ & 22 \\
\hline Prov. Buenos Aires $($ más de $50 \mathrm{~km})$ & 7 \\
\hline Santa Fe $(475 \mathrm{~km})$ & 4 \\
\hline Córdoba $(710 \mathrm{~km})$ & 3 \\
\hline Tucumán $(1.200 \mathrm{~km})$ & 1 \\
\hline Salta $(1.508 \mathrm{~km})$ & 2 \\
\hline Misiones $(1.005 \mathrm{~km})$ & 1 \\
\hline La Rioja $(810 \mathrm{~km})$ & 1 \\
\hline Formosa $(930 \mathrm{~km})$ & 2 \\
\hline Uruguay $(200 \mathrm{~km})(*)$ & 1 \\
\hline EE. UU. $(10.000 \mathrm{~km})(*)$ & 1 \\
\hline Totales & $\mathbf{9 8}$ \\
\hline
\end{tabular}

(*) Alumnos residentes fuera de Argentina 
El Grupo se configuró con una población de 98 docentes, en su mayoría femenino $(74,48 \%)$, con alta experiencia docente (edad promedio: 47,1 años), donde un porcentaje relativamente poco significativo (32\%) dijo usar estas innovaciones tecnológicas para aplicaciones personales. A su vez, casi las dos terceras partes del grupo $(61,6 \%)$ dijeron estar relativamente poco entrenados en informática (menos de 50 horas de entrenamiento). Los cursantes mostraron, en general, actitudes positivas hacia el uso de innovaciones tecnológicas (el 63,4\% mostró puntuaciones medias a altas en la medición de actitud), especialmente los de sexo masculino. La distribución por edades del grupo es significativa en términos de motivación y requerimientos de capacitación informática (Tabla $\mathrm{N}^{\circ} 2$ ).

\section{Tabla No 2: Distribución por edades de los grupos de alumnos}

\begin{tabular}{|l|c|}
\hline Edades entre & Alumnos \\
\hline 20 y 29 & 1 \\
\hline 30 y 39 & 24 \\
\hline 40 y 49 & 31 \\
\hline 50 y 59 & 34 \\
\hline 60 ó más & 5 \\
\hline Edad promedio: & 47,1 años \\
\hline
\end{tabular}

\section{Tutorías}

En Enseñanza a Distancia, el proceso de enseñanza-aprendizaje presenta algunas dificultades específicas. Distintos autores han tratado de explicar y compaginar la interacción -necesaria en todo proceso educativo- con la reducción de la presencialidad, típica de la Enseñanza a Distancia (Garcia Aretio, L.,1996). Todos los autores insisten en los siguientes aspectos:

1) animar a los alumnos para que sean activos,

2) aumentar la comunicación entre alumnos y tutores,

3) aumentar la retroalimentación, feedback, entre tutores y estudiantes, y

4) aumentar las oportunidades de interacción entre tutores y alumnos.

\section{Tutorías presenciales, telefónicas y correo electrónico} (Tabla $\mathrm{N}^{\mathrm{o}} 3$ ):

Este Curso de Postgrado contó con un apoyo tutorial, que se ofreció en tres modalidades

1) tutorías presenciales, optativas, durante el Curso.

tarde,

2) tutoría telefónica, dos días a la semana; uno, durante la mañana, de 9 a 13 horas, y otro, de de 14 a 18 horas, $y$

3) tutoría telemática, durante todos los días, en las veinticuatro horas. 


\section{Tabla $N^{0}$ 3: Tutorías realizadas en el primer cuatrimestre del curso PGEAD 1999}

\begin{tabular}{|c|c|cl|c|c|}
\hline Correo electrónico & Telefónica & Presencial & Fechas & Total & Porcentaje \\
\hline 10 & 6 & 0 & Desde el 10 de Abril & 16 & $16 \%$ \\
\hline 18 & 4 & 1 & Mayo & 23 & $23 \%$ \\
\hline 13 & 8 & 7 & Junio & 28 & $28 \%$ \\
\hline 18 & 8 & 5 & Al 15 de Julio & 31 & $31 \%$ \\
\hline $\mathbf{5 9}$ & \multicolumn{2}{|c|}{39} & & $\mathbf{9 8}$ & \\
\hline
\end{tabular}

La fecha de iniciación de las actividades fue el 10 de abril de 1999. La operadora de la Página Web fue la C.C. Estela Noemí Muelas.

\section{Tutoría telemática}

Los prerrequisitos que se tuvieron en cuenta, en función del entorno tecnológico de aprendizaje $\mathrm{y}$ de las necesidades del tutelado telemático, fueron:

1) Que el alumno posea una computadora con modem y línea telefónica, o tenga fácil acceso a ello y

2) que el alumno posea correo electrónico -E-mail- y conexión a Internet, o tenga fácil acceso a ello.

El grado de experiencia de los alumnos en la utilización de sistemas de comunicación por computadora no era parámetro limitante del curso. La tutoría telemática se basó en la Página Web del Curso y en el material didáctico específico, que posibilitó no solo la tutoría sino también la intercomunicación entre los alumnos. La iniciación de esta modalidad tutorial y la utilización de la herramienta telemática se realiza a partir del momento en que el alumno se familiariza con la comunicación electrónica. La operatoria del sistema es muy sencilla y el acceso a la Página Web y al conjunto de los materiales que la integran, está desarrollado de manera que la búsqueda de la información requerida -tanto "bajarla" ("download": "bajar" programas, o archivos, desde otra computadora), como la posibilidad de "levantarla" ("uploads": "subir" programas, o archivos, desde otra computadora)- documentación o mensajes, se realizan optimizando tiempos de comunicación.

Con respecto a la información disponible, la Página Web permite:

1) consultas, que se ponen a disposición del alumno en distintos tipos de documentos, textos, bases de datos, gráficos, etc.,

2) área de programas, donde el alumno puede recibir el software que necesite,

3) área de mensajes, dividida por temas, donde se contestan las preguntas y cuestiones que cualquier alumno pueda plantear, y

4) boletines y noticias, donde se informa a los alumnos de los aspectos relacionados con el Curso.

14 - Universidad ORT Uruguay 
Con este sistema, el alumno se puede comunicar y acceder a toda la información que necesita, operando desde su computadora con modem, y vinculándose, informáticamente, con los tutores y con los otros alumnos. El alumno puede utilizar cualquier modelo de computadora, de sistema operativo y de modem. El tutor, o el alumno, se pueden comunicar en cualquier momento, dejando al usuario la elección del momento más adecuado. Generalmente se utilizaron comunicaciones diferidas en el tiempo asincrónicas- superando así los problemas de horario, aunque también pudieron organizarse comunicaciones simultáneas en el tiempo -sincrónicas-, es decir en tiempo real.

Los mensajes pueden enviarse a todos los participantes o solo a una persona. El sistema utilizado permite un registro permanente de lo que se va haciendo y diciendo, al que tienen acceso los participantes. A este registro se accede con una palabra clave-password-que permite la identificación de cada usuario y que, obviamente, es secreta y personal. Después, cada participante puede recoger en sus archivos privados los datos que más le interesen.

\section{EI Foro}

En la implementación de la experiencia pedagógica a la que se denominó Foro -propuesta de discusión telemática-, se buscaba:

- generar intercambios para la construcción de saberes,

- aportar información para conceptualizar los temas,

- argumentar, no polemizar,

- publicar contenidos pertinentes a los temas del curso.

Para participar en el Foro, se configuraron algunas reglas básicas:

1) los temas serían moderados y evaluados por los coordinadores. La finalidad de esta moderación era la de crear un ambiente de análisis y discusión amigable, sin vulnerar la libertad de expresión de ninguno de los participantes.

2) Como era una experiencia pedagógica, los moderadores se reservaban el derecho de no publicar aquellos contenidos propuestos por los alumnos que no respondieran estrictamente a los objetivos del foro. Por ello, estaba claramente prohibido: a) desviarse del tema propuesto, b) el uso de expresiones discriminatorias o lesivas, c) todo tipo de ataque personal contra participantes del debate, y d) incluir material ajeno en los mensajes, o publicitar productos o actividades de cualquier índole.

Dentro de este ámbito:

1) los temas eran propuestos por los alumnos, de acuerdo con los contenidos y la problemática del Curso,

2) los alumnos intercambiaban opiniones y fundamentos,

3) si era necesario, los docentes moderaban las intervenciones,

4) los temas se "cerraban" pasado determinado tiempo, y los docentes emitían sus conclusiones,

5) hubo una evaluación global de la participación y de los niveles de intervención de los alumnos.

\section{Participación de los alumnos en el Foro}

Sobre un total de 98 alumnos, intervinieron activamente -obviamente, con distintos niveles de participación- 65 alumnos. Los 14 temas que se "subieron" al Foro, ordenados por el número de intervenciones, fueron: 
- Medios Impresos (25)

- Necesidades del mercado y educación (21)

- Relación espacio virtual / real (18)

- EAD sin encuentros presenciales (14)

- Capacitación y nuevas tecnologías (10)

- El texto paralelo (9)

- Preparación para el cambio (7)

- Las funciones del proceso educativo (7)

- Interactividad (7)

- Crítica a la teoría de la industrialización (6)

- La EAD desde el enfoque socio-económico (6)

- Meta-análisis del curso (5)

- La EAD como respuesta a la realidad (5)

- Paradigma tecnológico de intervención pedagógica (3)

El número de participantes en el intercambio fue el siguiente (Tabla $\left.\mathrm{N}^{\circ} 4\right)$ :

\section{Tabla $N^{0}$ 4: Intervenciones de alumnos}

\begin{tabular}{|c|c|}
\hline Veces & Alumnos \\
\hline 1 & 21 \\
\hline 2 & 30 \\
\hline 3 & 33 \\
\hline 4 & 20 \\
\hline 5 & 15 \\
\hline 6 & 24 \\
\hline 7 & 0 \\
\hline 8 & 0 \\
\hline 9 & 27 \\
\hline 10 & 0 \\
\hline 11 & 11 \\
\hline 12 & 0 \\
\hline 13 & 26 \\
\hline
\end{tabular}


Es importante destacar que sobre los 98 alumnos inscriptos originariamente participaron, con mayor o menor intensidad, la mayoría de los 65 alumnos que terminaron el curso, con un total de 144 intervenciones, y un promedio de 2,21 intervenciones por alumno.

\section{Procesamiento de los datos}

Casi la totalidad del procesamiento de los datos se realizó con un programa de análisis estadístico de registros de servidor Web. Provee una interfase amigable desde la que se puede acceder a una cantidad importante de estadísticas, tablas y gráficos, que informan y representan la evolución de una Página Web desde diversas perspectivas, y permite al usuario obtener una visión completa de la evolución de la misma.

Este método facilita un variado menú de informes, útiles para analizar la evolución del sitio e imprescindibles para el desarrollo de nuevas secciones y profundización de las ya existentes, ya que permite conocer qué, quiénes y cómo están visitando el sitio.

\section{Generación de estadísticas}

El sitio Web de Fundec almacenó toda la actividad referida a los usuarios en una base de datos SQL. Sobre cada "hit" se registra fecha, hora, dirección IP del usuario, navegador, página requerida (incluyendo gráficos), referente y bytes transferidos. Los datos del "servidor" de la página permitieron a los tutores generar las estadísticas necesarias para "monitorear" el proceso, teniendo en cuenta distintas unidades de medida, períodos a analizar, tipo de información requerida, amplitud de la muestra y tipo de representación.

En lo que corresponde a unidades de medida, básicamente, la actividad del sitio puede medirse en hits -donde cada página o gráfico es un hit-o bytes - donde el tamaño de cada página o gráfico es tenido en cuenta-. Cada hit representa un pedido efectuado al servidor Web, mientras que los bytes representan la cantidad de información transferida. El tamaño de la muestra que se elija permite ampliar, o restringir, el alcance de los datos a visualizar, teniendo en cuenta, si se considera necesario, los valores más representativos. En nuestro caso, el «tamaño» de cada página o gráfico era de 4,63 a 5,49 Kb.

Así, pudieron obtenerse distintos formatos de representación, ya sean tablas con los valores correspondientes, o una representación gráfica de la información. De acuerdo con la estadística solicitada, el gráfico será de torta, de barras, o curvas. El programa permite restringir el período a analizar, teniendo en cuenta las siguientes opciones: todo (no efectúa limitación alguna); hoy (solo tiene en cuenta la actividad del sitio registrada después de las 0 horas del día de la fecha); últimas $\mathbf{2 4}$ horas (tiene en cuenta sólo la actividad del sitio de las últimas 24 horas; esta semana (la información de la actividad registrada en la semana en curso; últimos 7 días, este mes, últimos 30 días y este año, cumplen similar cometido, restringiendo la información de acuerdo con lo solicitado. También permite extender, o restringir, el tipo de dato a analizar, teniendo en cuenta las siguientes opciones: Navegador (se totalizan los accesos por navegador o "browser"), Host (agrupa la actividad de cada dirección de la red desde la que se visitó el sitio), Dominio (la actividad se agrupa por dominio: argentina, comercial, etc.), Página (se verifica la actividad por página del sitio), Referente Externo y/o Referente Interno (cada navegador registra, e informa al servidor, la página que el usuario visitó antes de la actual, información conocida como referente o "referer", y que es muy útil para conocer, en el caso del referente externo, desde qué sitios están derivando visitantes al nuestro, y cómo navegan los usuarios nuestro sitio, referente interno) (Tablas $\mathrm{N}^{\circ}$ $5,6$ y 7$)$. 


\section{Tabla No 5: Datos Generales}

\begin{tabular}{|l|r|r|r|r|}
\hline & abr & may & jun & jul \\
\hline Promedio de kb (datos) transferidos por día & 744 & 957 & 906 & 3459 \\
\hline Promedio de kb (datos) transferidos por HIT & 4.6 & 5.4 & 4.7 & 5.3 \\
\hline Promedio de kb (datos) transferido por usuario & 81.1 & 67 & 80.8 & 91.2 \\
\hline Promedio de HITs por día & 161.7 & 177.1 & 192.8 & 652.6 \\
\hline Promedio de HITs por usuario & 17.6 & 12.4 & 17.2 & 15.4 \\
\hline Promedio de usuarios por día & 9.1 & 14.2 & 11.2 & 37.9 \\
\hline Cada usuario visita aprox. & 2.2 veces & 4.3 & 3.4 & 9.2 \\
\hline Total HITs & 3882 & 5138 & 5401 & 13053 \\
\hline Períodos registrados & 24 días & 29 días & 28 días & 20 días \\
\hline Total de visitas usuarios & 220 & 414 & 314 & 758 \\
\hline
\end{tabular}

\section{Tabla $N^{0}$ 6: Pedidos de páginas (HITs) dentro de PGEAD}

\begin{tabular}{|l|c|c|c|r|}
\hline Páginas & abr & may & jun & jul \\
\hline Foro & 6 & 13 & 4 & 55 \\
\hline Interactividad (foro) & & 2 & 1 & 13 \\
\hline Informaciones & 4 & 4 & 3 & 61 \\
\hline Menú & 4 & 4 & 2 & 59 \\
\hline Módulo1 & 2 & & 1 & 14 \\
\hline Módulo2 & 2 & & 1 & 16 \\
\hline Módulo3 & 2 & & 1 & 14 \\
\hline Principal & 2 & 3 & 1 & 43 \\
\hline Cartelera & 2 & 6 & 1 & 42 \\
\hline Calendario & & & & 24 \\
\hline
\end{tabular}




\section{Tabla Nº 7: Días más visitados del mes}

\begin{tabular}{|c|c|r|r|r|r|r|r|}
\hline \multicolumn{2}{|c|}{ ABRIL } & \multicolumn{2}{|c|}{ MAYO } & \multicolumn{2}{c|}{ JUNIO } & \multicolumn{2}{|c|}{ JULIO } \\
\hline 13 & $65 \%$ & 16 & $16 \%$ & 20 & $39 \%$ & 1 & $17 \%$ \\
28 & $15 \%$ & 30 & $15 \%$ & 10 & $34 \%$ & 7 & $12 \%$ \\
29 & $11 \%$ & 13 & $13 \%$ & 17 & $13 \%$ & 2 & $11 \%$ \\
& & 12 & $12 \%$ & 6 & $13 \%$ & 6 & $10 \%$ \\
\hline
\end{tabular}

\section{Conclusiones}

Los elementos más destacados de la tutoría telemática fueron:

a) la tutoría, en sus distintos aspectos, y

b) el Foro.

\section{Evaluación de la tutoría}

El análisis de los datos del primer cuatrimestre permite caracterizar, en una primera instancia, las siguientes situaciones:

1) Se dio una participación creciente.

2) Las consultas se centraron en aspectos organizativos.

3) Los materiales no presentaron dificultades.

4) Las personas más activas en la Página y en el Foro también lo fueron en las otras vías tutoriales.

5) Fue lenta la incorporación del correo electrónico y de la página Web.

6) No hubo dificultades tecnológicas importantes.

7) Se alcanzó un ritmo adecuado recién a partir de junio.

8) La mayoría satisfizo las expectativas de logro del cuatrimestre.

9) Se requirió por teléfono información ya dada en la Página Web, o en la Guía.

10) La producción personal se inició, para muchos, a fines de junio.

11) La falta de experiencia en estudiar a distancia generó ansiedad acerca de la ejecución adecuada de las tareas.

\section{Evaluación del Foro}

El análisis de los datos del primer cuatrimestre también permite caracterizar, en una primera instancia, las siguientes situaciones generadas en el foro:

- Ingresaron inicialmente los alumnos con experiencia en esta modalidad.

- Al promediar el cuatrimestre hubo un gran incremento de participación. 
- La tutoría realizó una moderación previa a la publicación de los aportes.

- Culminó con un índice elevado de participación.

También cabe tener en cuenta los siguientes aspectos:

- No todos los textos se ajustaron al tipo de escritura requerido por el medio: claridad, síntesis, estilo, longitud, etc.

- Algunas veces se aportaron citas sueltas que no siempre implicaron ni planteo de problemas ni argumentos.

- Se enunciaron preguntas muy abiertas, generalmente difíciles de tratar.

\section{Puede decirse que el Foro:}

los intercambios.

- es un espacio abierto, muy poco regulado, que motiva a participar espontáneamente y facilita

- puede alcanzar poca profundidad, estancarse en la conceptualización, o diluirse y dispersarse en el tratamiento de los temas.

- es apropiado para grupos que se inician como tales, donde se requiere estimular la participación.

En esta experiencia, la tutoría trató de ser "invisible" para no inhibir la participación por miedo al error, y debe destacarse que el valor de esta experiencia reside en la naturalidad y en la escasez de restricciones.

\section{Conclusiones generales}

El análisis de los datos anteriores permite caracterizar, en esta instancia, cinco perfiles de alumnos:

1) los que simplemente se conectan y recorren el sistema sin darse a conocer: no buscan comunicarse sino simplemente "ver cómo es",

2) los que se conectan buscando información para "ver si hay novedades": estos alumnos tampoco tienen interés en comunicarse, solo buscan informarse,

3) los que buscan acrecentar su caudal de materiales y se conectan para "bajar archivos": tampoco tienen gran interés en comunicarse, sino solo buscan "nutrirse",

4) los que se conectan y se comunican con el tutor para satisfacer dudas especificas de conocimiento: se mueven en el modelo "alumno que pregunta-docente que responde", y

5) los que se conectan y establecen el diálogo: son los que se comunican genuinamente con los tutores y con los colegas compañeros de curso, tanto desde el punto de vista del estudio como del de los lazos sociales.

Estos diferentes estilos responden a la representación, o modelo mental, que el cursante haya podido construir acerca de la comunidad de aprendizaje virtual que el medio telemático le posibilita. En función de la experiencia como tutores, para promover la comunicación en una comunidad virtual de aprendizaje se recomienda:

1) Brindar respuestas inmediatas porque realimentan y estimulan el aprendizaje. con otros alumnos.

2) Personalizar los mensajes para implicar al alumno y, simultáneamente, inducir la vinculación 
3) Incorporar materiales complementarios -específicos en la tutoría telemática-para la tarea a desarrollar en cada asignatura.

4) Incrementar la participación, en la tutoría telemática, de los docentes autores de los materiales para enriquecer el intercambio de ideas.

5) Inducir a los alumnos a responder a las demandas de otros compañeros y apoyar el trabajo en equipos virtuales.

6) Ayudar, desde el enfoque del material didáctico provisto, a revisar las pautas de implicación de los alumnos y su disposición a comunicarse y al trabajo colaborativo.

En este tipo de experiencias a distancia, y en general en cualquier proceso instruccional, las competencias del receptor y la participación voluntaria en la situación no son los únicos factores que desencadenan buenos aprendizajes. También lo son la calidad de la interacción generada en el entorno de aprendizaje, la intervención pedagógica del docente y la "cultura" del grupo respecto del valor de la actividad. En definitiva, la calidad de la interacción determina la dinámica del proceso.

Queda claro que el nivel de interacción que un recurso informático logra, depende de múltiples factores, algunos intrínsecos al mismo y que tienen que ver con la calidad de su diseño, y otros relacionados con el receptor y las condiciones de la situación de aprendizaje. Si bien es cierto que hay recursos como los que aquí analizamos, interactivos "per se", es posible hablar de interacción en muchos sentidos:

1) el sujeto interactúa con diversos materiales y construye el mensaje, integrándolos,

2) el recurso brinda un mensaje complejo codificado en varios sistemas simbólicos simultáneos,

3) el sujeto modifica su estrategia en función de las respuestas que le brinda el recurso,

4) el recurso se modifica teniendo en cuenta la estrategia del sujeto.

La interacción, desde un enfoque psicológico y pedagógico integrado, es un concepto mucho más rico y abarcador, que sólo se verifica integralmente en un contexto comunicativo humano, al cual se integran recursos cada vez más promisorios si se los utiliza adecuadamente. Mejores recursos no implican necesariamente mejores aprendizajes. Sólo:

1) un buen diseño de la situación didáctica,

2) problemas pertinentes, bien planteados y estimulantes,

3) alumnos con las correspondientes competencias para abordarlos, y

4) recursos válidos en función de los objetivos propuestos, resuelven la "ecuación" de la calidad educativa.

Para analizar la eficacia de un determinado recurso informático en alcanzar un logro educativo específico, debemos tener muy en cuenta que no existen efectos directos y lineales, sino que los resultados dependen en gran medida de la interacción de las variables que afectan al proceso: el método de enseñanza, el ambiente de enseñanza, la actitud de los actores implicados, los logros anteriores, el estilo y nivel cognitivo de cada uno, etc.

A partir de un análisis detallado del comportamiento de los cursantes, se podría evaluar más integralmente la acción tutorial y el modelo de gestión del sistema. Esto permitiría introducir gradualmente modificaciones destinadas a promover los estilos más positivos de trabajo en los cursantes, y orientadas a lograr una interacción más plena y eficaz. También queremos destacar que preocupa menos la rápida evolución de las tecnologías que la lenta adaptación de lo pedagógico-didáctico tanto al diseño curricular 
como al diseño de material didáctico requerido por las nuevas situaciones de enseñanza generadas por la evolución tecnológica: ya se avizora Internet 2, con una transmisión de datos a $155 \mathrm{Mbps}$, y se habla de velocidades de transmisión de datos de 2,4 Gbps -2400 millones de bits por segundo- para los próximos años (lo que significa transmitir una enciclopedia de treinta volúmenes en menos de un segundo).

Lo que interesa saber es si esas tecnologías y esos medios pueden ser usados pedagógicamente. Hasta ahora, a Internet se la considera tanto un medio de comunicación como un medio educativo. Queda claro que es un medio de comunicación, pero para que se convierta en un medio educativo será necesario crear, o adaptar, nuevas configuraciones curriculares y diseñar material didáctico para estas nuevas situaciones de enseñanza-aprendizaje.

Si bien, tal como dijimos antes, los entornos tecnológicos significan un avance crucial en las potencialidades de la educación a distancia, también es cierto que dichos entornos exigen y ponen en juego en las personas habilidades cognitivas y metacognitivas enteramente nuevas, que los adultos actuales deben adquirir gradualmente para actuar eficazmente en la formación.

Los nuevos modos de gestionar la información y la introducción de entornos tecnológicos multimediales en educación, tanto en la educación en general como en la educación a distancia en particular, deben proponer a los alumnos el desarrollo de nuevas competencias, la puesta en juego de estrategias cognitivas y metacognitivas diferentes a las utilizadas en contextos educativos tradicionales y un abordaje de la comunicación diferente: tratar eficazmente la información en este paradigma implica poseer otras competencias.

Por ello, las competencias para gestionar el propio aprendizaje y el grado de motivación puestos en juego en la situación, por una parte, y los sistemas de símbolos utilizados en las herramientas informáticas, cada uno por sus propiedades intrínsecas y en función de cómo se los combine para la presentación del mensaje, parafraseando a $\mathrm{H}$. Lasswell, determinarían parcialmente quién adquiere, cuánto conocimiento, de qué clase de mensajes, y con qué profundidad de elaboración. Dentro de la modalidad Enseñanza a Distancia se establece una estrecha relación entre el qué y el cómo enseñar.

También nos quedan algunos interrogantes para orientar nuevas investigaciones:

1) ¿Cuáles son las barreras en la comunicación que genera el medio telemático que puedan afectar el potencial de interacción?

2) ¿Cómo adquirir confianza para comunicarse en un "espacio virtual y público" donde perdemos el control de cómo y quiénes reciben nuestros mensajes, al desvanecerse el contacto "cara a cara" y los signos no verbales que completan el significado?

3) ¿Qué capacidades y actitudes; desde lo cognitivo, afectivo y emocional; juegan en la consolidación de un ambiente de aprendizaje virtual? 


\section{Bibliografía}

Gallego, D.J. 1993 "La tutoría en la enseñanza a distancia" PATED-ANCED: 219-261.

García Aretio, L. 1996 La educación a distancia y la UNED. IUED-UNED. Madrid.

García Aretio, L. (coord.) 1997 Investigar para mejorar la calidad de la Universidad. IUED-UNED. Madrid.

Jones, A.; Kirkwood, A. y Kirkup, G. 1992 Personal Computers for Distance Learning. London: Chapman.

Marabotto, M.I. y Grau, J.E. 1995 Multimedios y educación. Buenos Aires: Fundec.

Marabotto, M.I. y Grau, J.E. 1999 "La tutoría telemática en la educación a distancia", en Revista Iberoamericana de Educación a Distancia (RIED) Nº 2 (1), páginas 117 a 138.

Robinson, J. 1989 Training for Impact. New York: Jossey Bass.

UNESCO 1998 Aprendizaje abierto y a distancia: perspectivas y consideraciones políticas. IUEDUNED. Madrid.

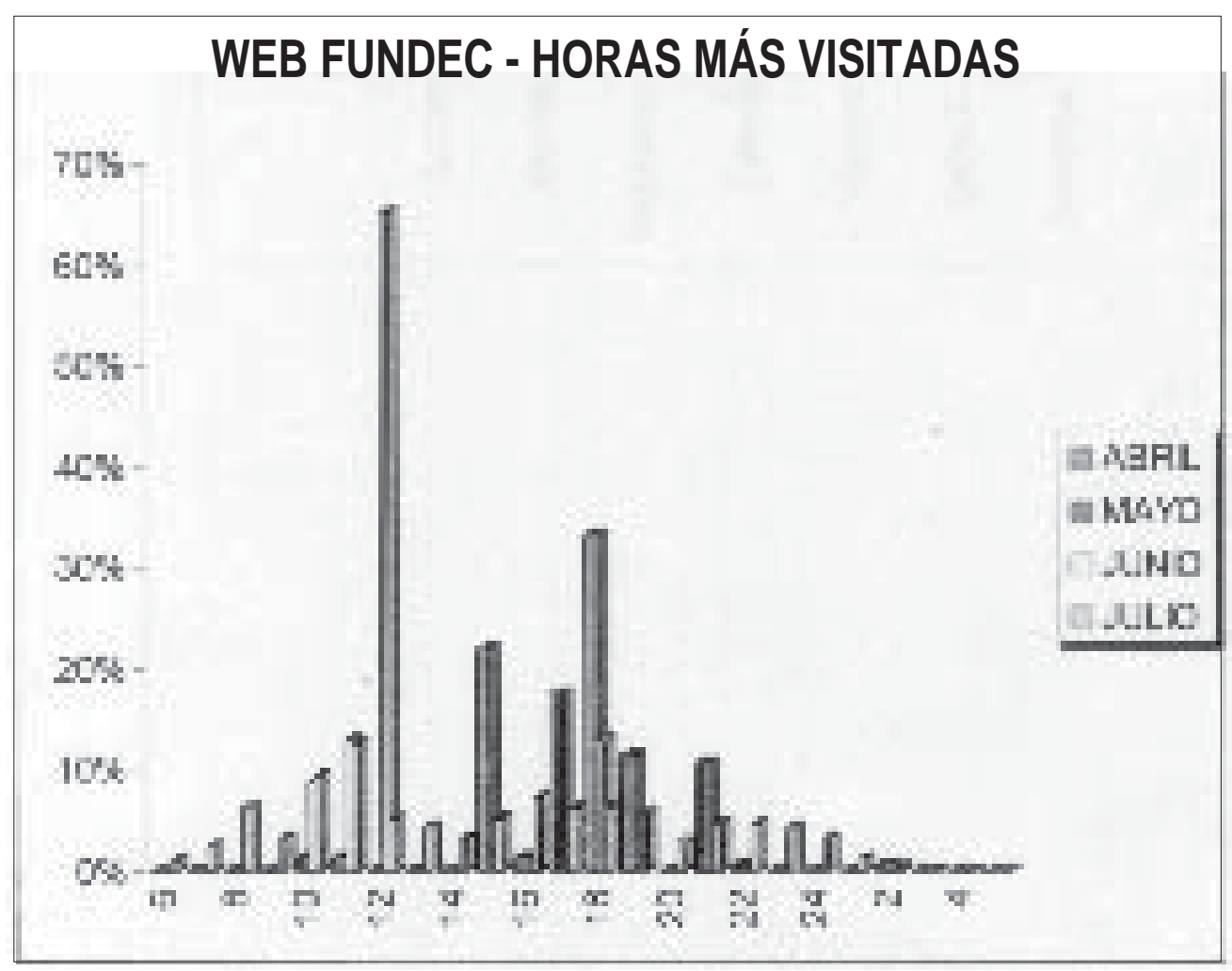




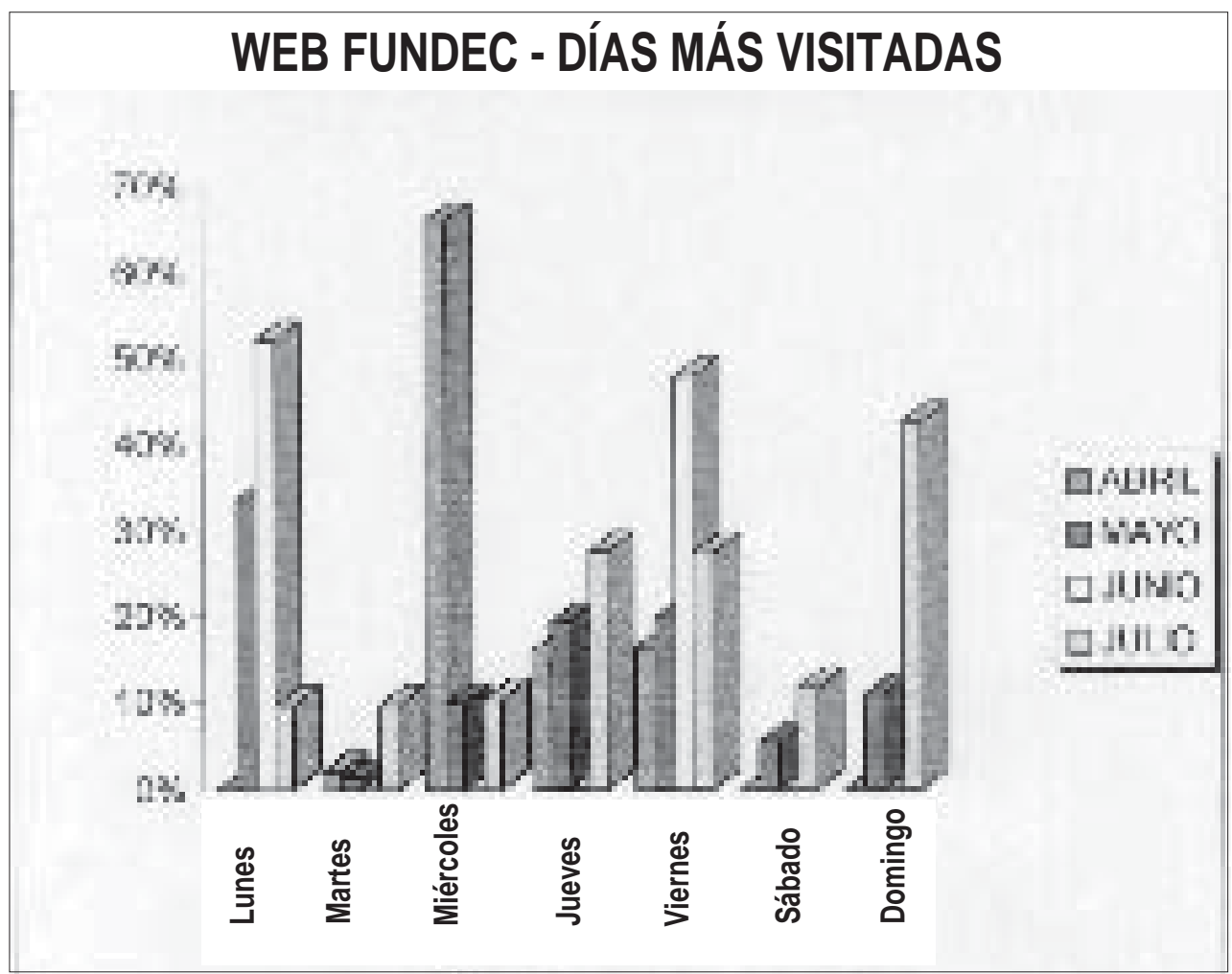

*, **, *** Universidad CAECE - FUNDEC - Buenos Aires - República Argentina 